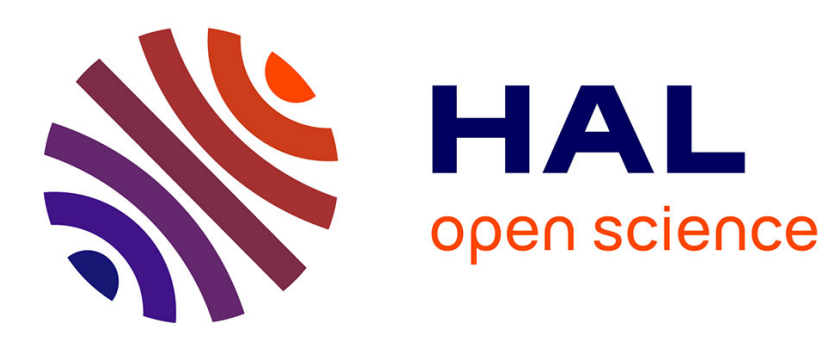

\title{
Optimal control for a self-reacting point absorber: A one-body equivalent model approach
}

Sébastien Olaya, Jean-Matthieu Bourgeot, Mohamed Benbouzid

\section{To cite this version:}

Sébastien Olaya, Jean-Matthieu Bourgeot, Mohamed Benbouzid. Optimal control for a self-reacting point absorber: A one-body equivalent model approach. IEEE PEAC 2014, IEEE, Nov 2014, Shanghai, China. pp.332-337, 10.1109/PEAC.2014.7037877 . hal-01121374

\section{HAL Id: hal-01121374 \\ https://hal.science/hal-01121374}

Submitted on 28 Feb 2015

HAL is a multi-disciplinary open access archive for the deposit and dissemination of scientific research documents, whether they are published or not. The documents may come from teaching and research institutions in France or abroad, or from public or private research centers.
L'archive ouverte pluridisciplinaire HAL, est destinée au dépôt et à la diffusion de documents scientifiques de niveau recherche, publiés ou non, émanant des établissements d'enseignement et de recherche français ou étrangers, des laboratoires publics ou privés. 


\section{Optimal Control for a Self-Reacting Point Absorber: A One-Body Equivalent Model Approach}

\author{
Sébastien Olaya \\ ENIB \\ EA 4325 LBMS \\ olaya@enib.fr
}

\author{
Jean-Matthieu Bourgeot \\ ENIB \\ EA 4325 LBMS \\ bourgeot@enib.fr
}

\author{
Mohamed Benbouzid \\ University of Brest \\ EA 4325 LBMS \\ Mohamed.Benbouzid@univ-brest.fr
}

\begin{abstract}
This paper deals with the optimal control of a selfreacting Wave Energy Converter (WEC) where the reaction force is obtained using a damping-plate. Model Predictive Control (MPC) is applied for unconstrained and constrained input control cases. Objective function attempting to optimise the power generation is directly formulated as an absorbed power maximisation problem and thus no optimal references, such as buoy and/or spar velocity, is required. Moreover, rather than using the full WEC model in the optimisation problem which can be timeconsuming, and because of linear assumptions, we propose the use of a phenomenologically one-body equivalent model derived using the Thévenin's theorem.

Index Terms-wave energy converter, phenomenologically onebody equivalent model, optimal control, model predictive control.
\end{abstract}

\section{INTRODUCTION}

This paper is about the development of a new french wave energy converter referenced as the "EM Bilboquet" project (Fig. 1). The power take-off (PTO) extracts mechanical power due to incoming waves by a system made up of a cylindrical buoy sliding along a partially submerged structure. This structure is made up of a vertical cylinder, referenced in the following as spar, with a damping plate attached at its keel. Energy resulting from the relative motion between the two concentric bodies is harnessed by rack-and-pinion which drives a permanent magnet synchronous generator through a gearbox. Wave energy converters using a reaction source which is not the seabed i.e. such as a plate, are referenced in the wave energy literature as a self-reacting WEC and because horizontal dimensions of the buoy is small compared to the incident wave length, the term of self-reacting point absorber is used. The use of a submerged body acting as a reference for the floating body which can react against is not a new concept but have a promising future.

Regarding the control strategy, it is well established now, that it is an important WEC design aspect to make it more efficient, and lot of efforts have been put on it during the last decade. Several strategies have already been proposed and can be classified in two main categories, namely passive and, what we will call in the following, active loading. Model Predictive Control (MPC) in the wave energy context belongs to the latter, and has already received most attention from wave energy converter community. In [1], Cretel studies optimal control for a single-body point absorber case with objective function

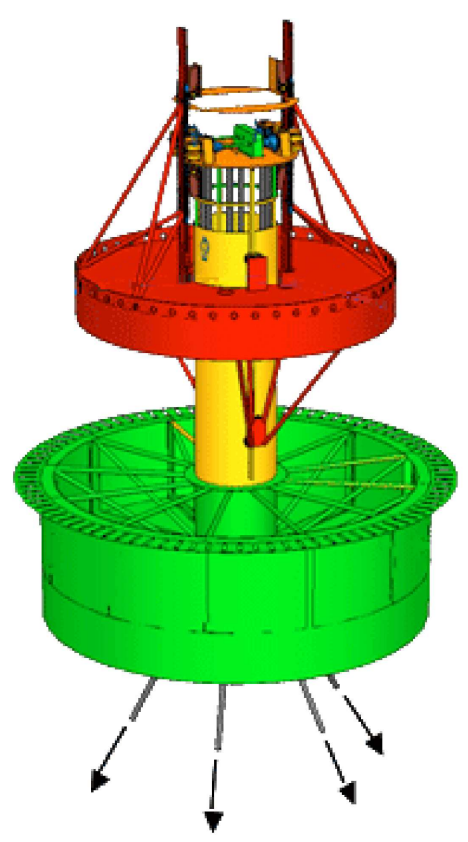

Fig. 1: Project "EM Bilboquet".

formulated on the absorbed energy. In [2], author presented design details and simulation results for a Model Predictive Control (MPC) for a two-body WEC. Objective function in the optimisation problem is formulated as a tracking error problem where reference is the optimal buoy velocity. However the used hydrodynamic WEC model was too simple in the fact that it did not take into account the model frequency dependence and it restricted the two-body dynamics to a single one assuming a perfect stationary reference body (the central spar is perfectly motion-damped either via mooring or using a submerged damping-plate). In [3], author completed the previous work by considering a linear two-body dynamic. In [4] the same authors proposed a non-linear MPC (NMPC) formulation for a two-body WEC, in case where a non-linear mooring-line term is added to the model. In both papers, a full WEC model including a full radiation force description, was used but only for plant simulation purpose. Indeed, optimal control was formulated, based on a direct energy formulation, on a reduced model where authors assumed constant inertia and damping 
terms, claiming that convolution terms in radiation forces are "fairly small compared to other external forces". However, authors indicate that, if required, a full WEC model could be used in MPC formulation, based on a linear state-space approximation. The main counterpart in doing this, is that the WEC model order would increase considerably, which could be a real problem for real-time evaluation of the optimisation problem.

In this paper, we propose to use the "phenomenologically one-body equivalent model" concept, firstly introduced in [5]. Based on the well-known Thévenin's theorem, we identify a one-body equivalent state-space model excited by an equivalent wave excitation force, that is useful for formulating the optimisation problem because of its low order.

\section{The Self-Reacting Point Absorber Model}

\section{A. Mathematical Background}

In this section we present the mathematical formulation of the linearised model for a generic self-reacting $\mathrm{WEC}^{1}$. For the sake of simplicity, the total structure dynamics is restricted to the heaving mode. Under the assumption of linear wave potential theory, the linearised motion equation for the two bodies is given by the Newton's second law ${ }^{2}$

$$
\left\{\begin{array}{l}
m_{1} \ddot{z}_{1}=f_{\mathrm{ex}, 1}+f_{\mathrm{r}, 11}+f_{\mathrm{r}, 21}+f_{\mathrm{gen}}+f_{\mathrm{s}, 1} \\
m_{2} \ddot{z}_{2}=f_{\mathrm{ex}, 2}+f_{\mathrm{r}, 22}+f_{\mathrm{r}, 12}-f_{\mathrm{gen}}+f_{\mathrm{s}, 2}+f_{\mathrm{drag}}
\end{array}\right.
$$

where $m_{1}$ and $m_{2}$ are respectively the mass of the buoy and the spar, $z_{i}$ is the body $i$ vertical displacement with respect to the equilibrium position. $f_{\mathrm{ex}, i}$ is the wave excitation force applied on body $i$. It can be expressed in the time-domain as

$$
f_{\mathrm{ex}, i}(t)=\int_{-\infty}^{\infty} h_{\mathrm{ex}, i}(t-\tau) \eta_{\mathrm{o}}(\tau) \mathrm{d} \tau
$$

where $\eta_{\mathrm{o}}(t)$ is the wave elevation due to the incident wave at the origin $\mathrm{O}$, located at the intersection of the undisturbed free surface level with cylinder axis and $h_{\mathrm{ex}, i}(t)$ is the impulse response of the wave excitation force related to the geometry of the body $i$ [6]. $f_{\mathrm{r}, i j}$ is the force applied on the body $j$ due to the motion of body $i$. This force is associated to the radiation problem. In linear potential theory, it is conventional to decompose this force in two parts which are frequency dependent. One is proportional to the body acceleration, the other is proportional to its velocity and, are respectively referenced as added mass and radiation damping

$$
f_{\mathrm{r}, i j}=-m_{\mathrm{a}, i j} \ddot{z}_{i}(t)-b_{i j} \dot{z}_{i}(t)
$$

Because of the hydrodynamic coefficient frequency dependence, it is convenient to replace (3) by an easiest computational formulation. Cummins [7] shown that it can be approximated by the following representation in the timedomain for the zero forward speed case

$$
f_{\mathrm{r}, i j}=-m_{\mathrm{a}, i j}(\infty)-\int_{-\infty}^{t} k_{i j}(t-\tau) \dot{z}_{i}(\tau) \mathrm{d} \tau
$$

\footnotetext{
1 i.e. we do not make any assumption on the PTO principle.

${ }^{2}$ Indices 1 is used for what is refereed to the buoy and 2 for the spar
}

where $m_{\mathrm{a}, i j}(\infty)$ is defined as the infinite added mass and $k_{i j}(t)$ is the radiation convolution kernel. $f_{\mathrm{s}, i}$ is the net restoring force due to gravity and buoyancy. It is proportional to the displacement of the body structure from its equilibrium position. The coefficient of proportionality is denoted $\kappa_{\mathrm{s}, i}$ and is referenced as the buoyancy stiffness

$$
f_{\mathrm{s}, i}(t)=-\kappa_{\mathrm{s}, i} z_{i}(t)
$$

where the diagonal elements are respectively defined for the buoy and the platform by $\kappa_{\mathrm{s}, 1}$ and $\kappa_{\mathrm{S}, 2}$ such as

$$
\kappa_{\mathrm{s}, i}=\rho g \iint_{S_{\mathrm{F} 0, i}} \mathrm{~d} S
$$

where $\rho$ is the water density, $g$ is the gravitational acceleration and $S_{\mathrm{F} 0, i}$ is the water plane area at equilibrium condition. In the cylindrical shape case, we have $\kappa_{\mathrm{s}, 1}=\rho g \frac{\pi}{4}\left(D_{\mathrm{b}}^{2}-D_{\mathrm{s}}^{2}\right)$ for the buoy and $\kappa_{\mathrm{s}, 2}=\rho g \frac{\pi}{4} D_{\mathrm{s}}^{2}$ for the spar. In order to enhance the spar modelling during the resonant oscillation we use, as a first approximation, a constant linear damping term $b_{\text {drag, }}$, proportional to the spar velocity such as $f_{\text {drag }}=$ $-b_{\text {drag }} \dot{z}_{2}$. In this paper the additional damping is chosen in such a way that it corresponds to the maximum dissipation of the non-linear term [8] when optimal active control is applied $^{3}$ which corresponds approximatively, after numerical investigation, to $14.5 \%$ of the critical damping defined as $b_{\text {crit }, 2}=2 \sqrt{\left(m_{2}+m_{\mathrm{a}, 22}(\infty)\right) \kappa_{2}}$. Finally, $f_{\text {gen }}$ denotes the force due to the generator which is also the control input. One could include additional restoring or non-linear forces due to mooring lines. However in the following, we would assume that the energy extraction in heaving mode is not or less-perturbed by this effort.

\section{B. Approximated State-Space Model}

Based on the above development and using a matrix notation, the equation system (1) can be rewrite as

$$
\begin{aligned}
\left(\mathrm{M}+\mathrm{M}_{\mathrm{a}}(\infty)\right) \ddot{\xi}(t)+\int_{-\infty}^{t} \mathrm{~K}(t-\tau) \dot{\xi}(\tau) \mathrm{d} \tau \\
\quad+\mathrm{B}_{\mathrm{drag}} \dot{\xi}(t)+\mathrm{K}_{\mathrm{s}} \xi(t)=F_{\text {ex }}(t)+F_{\text {gen }}(t)
\end{aligned}
$$

where $\xi=\left[\begin{array}{ll}z_{1} & z_{2}\end{array}\right]^{T}$. This integro-differential equation is referenced in the literature as the Cummins formulation. It is well established in the wave energy community that direct computation of (7), based on a discrete-time approximation, is not efficient and not appropriated for control purposes. The use of parametric models based on a state-space representation that approximate the convolution kernels (2) and (4) are more suitable. In [9], authors provide a MATLAB toolbox which approximate the convolution terms of (4) by a linear timeinvariant system. Regarding the wave excitation forces, Falnes (1995), in [10], shown that the convolution kernel $h_{\mathrm{ex}, i}(t)$ of (2) is not necessary causal because of the mathematical assumptions made for the hydrodynamic parameter determination.

\footnotetext{
${ }^{3}$ Optimal active impedance is found in the frequency-domain by numerical exhaustive search based on the non-linear model.
} 


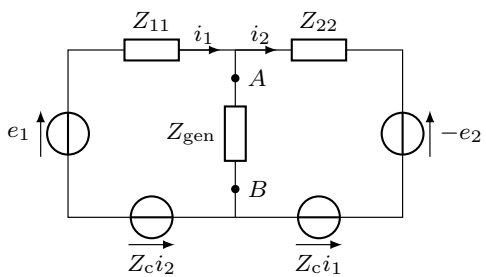

(a)

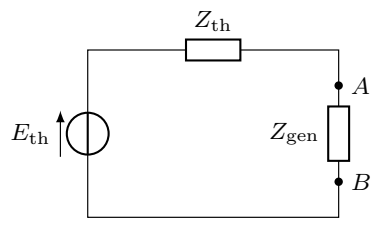

(b)
Fig. 2: Thévenin's equivalent model.

Hydrodynamic coefficients, i.e. added mass, radiation damping, and wave excitation force, that are required in the identification process, are computed in the frequency-domain by a semi-analytical method described in [11] and also in [12].

\section{Phenomenologically One-Body Equivalent MODEL}

\section{A. From a Frequency Domain Analysis...}

Assuming a harmonic decomposition, i.e. $\xi(t)=\hat{\xi} \exp ^{-\mathrm{i} \omega t}$, the equation system (1) can be rewrite in the frequency-domain in a matrix form such as

$$
\left[\mathrm{B}(\omega)+\mathrm{B}_{\text {drag }}+\mathrm{i} \omega\left(\mathrm{M}+\mathrm{M}_{\mathrm{a}}(\omega)-\frac{\mathrm{K}_{\mathrm{s}}}{\omega^{2}}\right)\right] \hat{\dot{\xi}}=\hat{F}_{\text {ex }}+\hat{F}_{\text {gen }}
$$

In [5], Falnes presents a phenomenologically one-body equivalent model for a self-reacting wave energy converter working in heaving mode and using the relative motion between the two bodies to extract power. Using the complex intrinsic mechanical impedance concept defined as

$$
Z_{i j}=\left[b_{i j}(\omega)+b_{\mathrm{drag}, i j}\right]+\mathrm{i} \omega\left[m_{i j}+m_{\mathrm{a}, i j}(\omega)-\frac{k_{\mathrm{s}, i j}}{\omega^{2}}\right]
$$

and assuming $\hat{f}_{\text {gen }}=Z_{\text {gen }}\left(\hat{\dot{z}}_{1}-\hat{\dot{z}}_{2}\right)$, without loss of generality, and $Z_{\mathrm{c}}=Z_{12}=Z_{21}$, we may rewrite (8) as

$$
\left[\begin{array}{cc}
Z_{11}+Z_{\text {gen }} & Z_{\mathrm{c}}-Z_{\text {gen }} \\
Z_{\mathrm{c}}-Z_{\text {gen }} & Z_{22}+Z_{\text {gen }}
\end{array}\right]\left[\begin{array}{l}
\hat{\dot{z}}_{1} \\
\hat{\dot{z}}_{2}
\end{array}\right]=\left[\begin{array}{l}
\hat{f}_{\text {ex }, 1} \\
\hat{f}_{\text {ex }, 2}
\end{array}\right]
$$

Using an electrical analogy i.e. $\hat{\dot{z}}_{i} \rightarrow i_{i}$ and $\hat{f}_{\mathrm{ex}, i} \rightarrow e_{i}$, see Fig. 2, and applying the well-known Thévenin's theorem for dependent sources, we found that the complex relative velocity $\hat{\dot{z}}_{\mathrm{r}}=\left(\hat{\dot{z}}_{1}-\hat{\dot{z}}_{2}\right) \rightarrow\left(i_{1}-i_{2}\right)$ can be expressed as

$$
\hat{\dot{z}}_{\mathrm{r}}=\frac{1}{Z_{\mathrm{i}, \mathrm{eq}}}\left(\hat{f}_{\mathrm{ex}, \mathrm{eq}}+\hat{f}_{\mathrm{gen}}\right)
$$

where $Z_{\mathrm{i} \text {,eq }} \rightarrow Z_{\mathrm{th}}$ is the intrinsic mechanical impedance for the one-body equivalent system and $\hat{f}_{\text {ex } \text { eq }} \rightarrow E_{\text {th }}$ is the complex equivalent wave excitation force, respectively defined as

$$
\begin{gathered}
Z_{\mathrm{i}, \mathrm{eq}}=\frac{Z_{11} Z_{22}-Z_{\mathrm{c}}{ }^{2}}{Z_{0}} \\
\hat{f}_{\mathrm{ex}, \mathrm{eq}}=\hat{f}_{\mathrm{ex}, 1} \frac{\left(Z_{2}+Z_{\mathrm{c}}\right)}{Z_{0}}-\hat{f}_{\mathrm{ex}, 2} \frac{\left(Z_{1}+Z_{\mathrm{c}}\right)}{Z_{0}}
\end{gathered}
$$

with

$$
Z_{0}=Z_{1}+Z_{2}+2 Z_{\mathrm{c}}
$$

The obtained equivalent model is quite useful for discussing optimum performance in regular wave analysis. Indeed it can be shown that the maximum absorbed power, when reactive control strategy ${ }^{4}$ is applied and no constraints are considered, is given by (15) [5], [13]

$$
Z_{\text {gen }, \text { opt }}=Z_{\mathrm{i}, \text { eq }}{ }^{*}
$$

where ${ }^{*}$ symbol denotes complex-conjugate.

\section{B. ... to a Phenomenologically One-Body Equivalent State- Space Model}

Equation (11) describes the equivalent wave excitation force to relative velocity relation. If we are able to identify a timedomain model based on data provided by this equivalent model, we could use it as it will be explained in the next section for formulating the control problem. In the timedomain, (11) can be expressed as a convolution product

$$
\dot{z}_{\mathrm{r}}(t)=\int_{-\infty}^{\infty} h(t-\tau)(u(\tau)+w(\tau)) \mathrm{d} \tau
$$

where $h(t)=\mathcal{F}^{-1}\left\{H(\mathrm{i} \omega) \equiv Z_{\mathrm{i}, \mathrm{eq}}{ }^{-1}\right\}, u(t) \equiv f_{\text {gen }}(t)$ and $w(t)$ is the equivalent wave excitation force in the time-domain defined as

$$
w(t)=\int_{-\infty}^{+\infty} g(t-\tau) \eta_{\mathrm{o}}(\tau) \mathrm{d} \tau
$$

where $g(t)$ is the inverse Fourier transform of $G(\mathrm{i} \omega) \equiv \hat{f}_{\text {ex }, \text { eq }}$

$$
g(t)=\frac{1}{2 \pi} \int_{-\infty}^{\infty} G(\mathrm{i} \omega) \exp ^{-\mathrm{i} \omega t} \mathrm{~d} \omega
$$

Two transfer functions have to be determined for having a full description of the equivalent one-body model. One is related to the force to relative velocity behaviour whereas the second is related to the wave surface elevation to equivalent wave excitation force behaviour. In this paper we focus our talk only one the identification of $H(\mathrm{i} \omega)$. A similar procedure than the one described below can be used for identifying $g(t)$. However, we have to consider a causal version of $g(t)$ in the identification process, and then the equivalent wave excitation force is provided by the following model

$$
w(t)=\int_{0}^{t+t_{\mathrm{c}}} g^{(\mathrm{c})}(t-\tau) \eta_{\mathrm{o}}\left(\tau+t_{\mathrm{c}}\right) \mathrm{d} \tau
$$

Because relative velocity is necessary a causal process, we used the Laplace formalism and then we look for an approximated transfer function such as

$$
\tilde{H}(s)=\frac{P(s)}{Q(s)}=\frac{p_{r} s^{r}+p_{r-1} s^{r-1}+\cdots+p_{0}}{s^{n}+q_{n-1} s^{n-1}+\cdots+q_{0}}
$$

Identification procedure is similar to the one presented in [14]. Convolution kernel properties of $h(t)$ are synthesised for both frequency- and time-domain in TABLE I. Once the model

\footnotetext{
${ }^{4}$ In case where optimal passive loading is applied, it can be shown that we have $R_{\text {gen,opt }}=\left|Z_{\text {i,eq }}\right|$
} 
TABLE I: Convolution Kernel $h(t)$ Properties

\begin{tabular}{ll}
\hline Properties & Implication on parametric models \\
\hline (1) $\lim _{\omega \rightarrow 0} H(\mathrm{i} \omega)=0$ & There are a zero at $s=0$ \\
$(2) \lim _{\omega \rightarrow \infty} H(\mathrm{i} \omega)=0$ & Strictly proper \\
$(3) \lim _{t \rightarrow 0^{+}} h(t) \neq 0$ & Relative degree is 1 \\
$(4) \lim _{t \rightarrow \infty} h(t)=0$ & BIBO stable \\
\hline
\end{tabular}

have been identified, an equivalent state-space model of (20) is directly given in a canonical form by

$$
\left\{\begin{array}{l}
\dot{x}(t)=A_{\mathrm{c}} x(t)+B_{\mathrm{c}}(u(t)+w(t)) \\
\dot{z}_{\mathrm{r}}(t) \simeq C_{\mathrm{c}} x(t)
\end{array}\right.
$$

where

$$
A_{\mathrm{c}}=\left[\begin{array}{ccccc}
-q_{n-1} & -q_{n-2} & \ldots & -q_{1} & -q_{0} \\
1 & 0 & \ldots & 0 & 0 \\
0 & 1 & 0 & \vdots & 0 \\
\vdots & \vdots & \ddots & \vdots & \vdots \\
0 & 0 & \ldots & 1 & 0
\end{array}\right], \quad B_{\mathrm{c}}=\left[\begin{array}{c}
1 \\
0 \\
\vdots \\
0 \\
0
\end{array}\right]
$$

and

$$
C_{\mathrm{c}}=\left[\begin{array}{lllll}
p_{r} & p_{r-1} & \cdots & p_{1} & p_{0}
\end{array}\right], \quad D_{\mathrm{c}}=0
$$

Figure 3 presents frequency response for the equivalent wave excitation force to relative velocity transfer function and for the wave surface elevation to equivalent wave excitation force. A $6^{\text {th }}$ order model have been found for the former and a $8^{\text {th }}$ order, with a $5[\mathrm{~s}]$ causalising time-shift $t_{\mathrm{c}}$, for the latest seems to be good enough.

It is important to mention there, that one can see the most advantage of using the phenomenologically one-body equivalent model for control purpose. Indeed, a $6^{\text {th }}$ order model is enough to fully describe the equivalent wave excitation force to relative velocity relation whereas at least a $24^{\text {th }}$ order would be necessary if the two-body dynamic would be considered. This will considerably decrease the complexity and the time evaluation of the control strategy presented in the next section.

\section{Optimal WEC CONTROL}

\section{A. Optimal Control Formulation}

The control objective is to maximise the absorbed energy $E_{\text {abs }}$ over a certain prediction horizon $T_{\text {hor }}$

$$
E_{\text {abs }}=-\int_{t}^{t+T_{\mathrm{hor}}} u(\tau) \dot{z}_{\mathrm{r}}(\tau) \mathrm{d} \tau
$$

where $\dot{z}_{\mathrm{r}}(t)$ is the relative velocity between the buoy and the spar such as $\dot{z}_{\mathrm{r}}(t)=\dot{z}_{1}(t)-\dot{z}_{2}(t)$. Using a rectangle rule integration method, this can be rewrite as

$$
E_{\mathrm{abs}} \simeq-T_{\mathrm{s}} \sum_{i=0}^{N_{\mathrm{hor}}} u_{k+i-1} \dot{z}_{\mathrm{r}, k+i}
$$
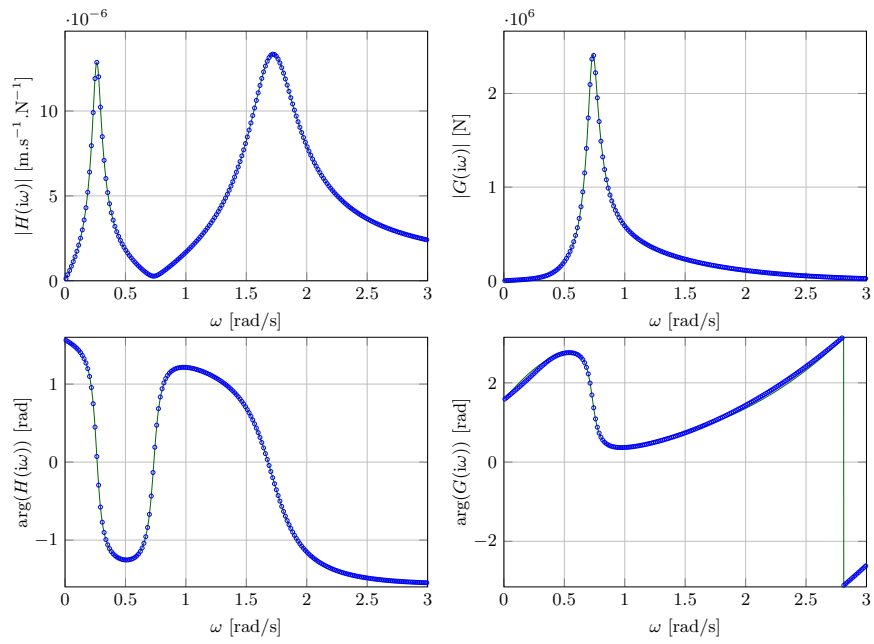

- hydrodynamic data _ estimated data

Fig. 3: Amplitude and phase of the frequency response for the equivalent one-body model.

where $T_{\mathrm{s}}$ is the sampling period and $N_{\text {hor }}=T_{\text {hor }} / T_{\mathrm{s}}$ denotes the prediction horizon length. Assuming a zero-hold order sampling, the one-body equivalent discrete state-space model (21) can be expressed as

$$
\left\{\begin{aligned}
x_{k+1} & =A_{\mathrm{d}} x_{k}+B_{\mathrm{d}}\left(u_{k}+w_{k}\right) \\
\dot{z}_{\mathrm{r}, k} & =C_{\mathrm{d}} x_{k}
\end{aligned}\right.
$$

and the control input equation is given by

$$
u_{k}=u_{k-1}+\Delta u_{k}
$$

Defining $\bar{x}_{k}=\left[\begin{array}{ll}x_{k} & u_{k-1}\end{array}\right]^{T}$ and considering $\bar{y}_{k}=$ $\left[\begin{array}{ll}\dot{z}_{\mathrm{r}, k} & u_{k-1}\end{array}\right]^{T}$, we can define the augmented system such ass

$$
\left\{\begin{aligned}
\bar{x}_{k+1} & =\left[\begin{array}{cc}
A_{\mathrm{d}} & B_{\mathrm{d}} \\
0 & 1
\end{array}\right] \bar{x}_{k}+\left[\begin{array}{c}
B_{\mathrm{d}} \\
1
\end{array}\right] \Delta u_{k}+\left[\begin{array}{c}
B_{\mathrm{d}} \\
0
\end{array}\right] w_{k} \\
\bar{y}_{k} & =\left[\begin{array}{ll}
C_{\mathrm{d}} & 0 \\
0 & 1
\end{array}\right] \bar{x}_{k}
\end{aligned}\right.
$$

or in a simple form

$$
\left\{\begin{aligned}
\bar{x}_{k+1} & =A \bar{x}_{k}+B_{u} \Delta u_{k}+B_{w} w_{k} \\
\bar{y}_{k} & =C \bar{x}_{k}
\end{aligned}\right.
$$

The absorbed energy function (23) can then be reformulated as

$$
E_{\mathrm{abs}} \simeq-T_{\mathrm{s}} \frac{1}{2} \sum_{i=0}^{N_{\mathrm{hor}}} \bar{y}_{k+i \mid k}^{T} Q \bar{y}_{k+i \mid k}
$$

with $Q=\left[\begin{array}{ll}0 & 1 \\ 1 & 0\end{array}\right]$ and where notation $k+i \mid k$ means the prediction at the time $k+i$ based on the information available at the instant $k$. 


\section{B. Model Predictive Control Strategy}

Maximising the absorbed energy (28) is equivalent to minimising

$$
J^{\prime}=\frac{1}{2} \sum_{i=0}^{N_{\text {hor }}}\left(\bar{x}_{k+i \mid k}^{T} \bar{Q} \bar{x}_{k+i \mid k}+\Delta u_{k+i \mid k}^{T} R \Delta u_{k+i \mid k}\right)
$$

where $\bar{Q}=C^{T} Q C$ and where we have introduce a cost penalty $R>0$ on the control input increment $\Delta u_{k}$. In (29), the first term $\bar{x}_{k \mid k}^{\mathrm{T}} \bar{Q} \bar{x}_{k \mid k}$ does not contribute to the optimal solution as the last term $\Delta u_{k+N_{\text {hor }} \mid k}^{T} R \Delta u_{k+N_{\text {hor } \mid k}}$ can be neglected because it does not contribute to the energy absorption over the prediction horizon length. Then (29) can be re-written such as

$$
\begin{aligned}
J\left(\Delta u_{k}\right)=\frac{1}{2} \sum_{i=1}^{N_{\text {hor }}}\left(\bar{x}_{k+i \mid k}^{T} \bar{Q} \bar{x}_{k+i \mid k}\right. & \\
& \left.+\Delta u_{k+i-1 \mid k}^{T} R \Delta u_{k+i-1 \mid k}\right)
\end{aligned}
$$

or in a matrix notation form

$$
J\left(\boldsymbol{\Delta} u_{k}\right)=\frac{1}{2} \bar{X}_{k}^{T} \mathbf{Q} \bar{X}_{k}+\frac{1}{2} \boldsymbol{\Delta} u_{k}^{T} \mathbf{R} \boldsymbol{\Delta} u_{k}
$$

where $\mathbf{Q}=\operatorname{diag}(\overbrace{\bar{Q}, \ldots, \bar{Q}}^{N_{\text {hor }}}), \mathbf{R}=\operatorname{diag}(\overbrace{R, \ldots, R}^{N_{\text {hor }}})$ and,

$$
\bar{X}_{k}=\left[\begin{array}{c}
\bar{x}_{k+1 \mid k} \\
\bar{x}_{k+2 \mid k} \\
\vdots \\
\bar{x}_{k+N_{\mathrm{hor} \mid k}}
\end{array}\right], \quad \Delta u_{k}=\left[\begin{array}{c}
\Delta u_{k \mid k} \\
\Delta u_{k+1 \mid k} \\
\vdots \\
\Delta u_{k+N_{\mathrm{hor}}-1 \mid k}
\end{array}\right]
$$

$\Delta u_{k}$ is the control sequence of $\Delta u_{k}$ over the prediction horizon length $N_{\text {hor }}$. When the state and/or the control input are subject to constraints, the optimisation problem can be formulated as

$$
\begin{array}{cl}
\underset{\Delta u_{k}}{\operatorname{minimise}} & J\left(\boldsymbol{\Delta} u_{k}\right) \\
\text { subject to } & u_{k} \in \mathbb{U}, k=1, \ldots, N_{\text {hor }} \\
& \bar{x}_{k} \in \mathbb{X}, k=1, \ldots, N_{\text {hor }}
\end{array}
$$

Model predictive control is based on the receding horizon concept. In a receding horizon framework, optimal control sequence $\Delta u_{k}^{*}$ is obtained when solving (32) at the current time $k$ but only the first optimal computed increment $\Delta u_{k \mid k}^{*}$ is applied.

$$
\Delta u_{k \mid k}^{*}=[1,0, \ldots, 0] \Delta u_{k}^{*}
$$

At the next sampling period the process is repeated with new measurements and estimations from the plant. From (27) and using notations previously introduced, we can define the state prediction equation over the horizon $N_{\text {hor }}$ as

$$
\bar{X}_{k}=J_{x} \bar{x}_{k \mid k}+J_{u} \boldsymbol{\Delta} u_{k}+J_{w} W_{k}
$$

where

$$
\begin{gathered}
J_{x}=\left[\begin{array}{c}
A \\
A^{2} \\
\vdots \\
A^{N_{\mathrm{hor}}}
\end{array}\right], \quad W_{k}=\left[\begin{array}{c}
w_{k \mid k} \\
w_{k+1 \mid k} \\
\vdots \\
w_{k+N_{\mathrm{hor}}-1 \mid k}
\end{array}\right], \\
J_{j}=\left[\begin{array}{cccc}
B_{j} & 0 & \cdots & 0 \\
A B_{j} & B_{j} & \cdots & 0 \\
\vdots & \vdots & \ddots & \vdots \\
A^{N_{\mathrm{hor}}-1} B_{j} & A^{N_{\mathrm{hor}}-2} B_{j} & \cdots & B_{j}
\end{array}\right]
\end{gathered}
$$

with $j \in\{u, w\}$. It is important to mention that (34) is only dependent on the current state $\bar{x}_{k \mid k}$ and on the equivalent wave excitation force prediction. Substituting (34) in (31) and omitting terms that do not depend of the control input increment sequence $\boldsymbol{\Delta} u_{k}$, optimisation problem cost function (31) can be rewritten in a quadratic form such as

$$
J\left(\boldsymbol{\Delta} u_{k}\right)=\frac{1}{2} \boldsymbol{\Delta} u_{k}^{T} H \boldsymbol{\Delta} u_{k}+f^{T} \boldsymbol{\Delta} u_{k}
$$

with

$$
\left\{\begin{array}{l}
H=J_{u}^{T} \mathbf{Q} J_{u}+\mathbf{R} \\
f=J_{u}^{T} \mathbf{Q}\left(J_{x} \bar{x}_{k \mid k}+J_{w} W_{k}\right)
\end{array}\right.
$$

By inspection, we find that the Hessian matrix $H$ is positive definite hence optimisation problem (32) has a unique solution and can be efficiently solved using quadratic programming.

\section{Numerical Results For Optimal Control}

In this section, we present numerical results for both, regular and irregular wave conditions. All time-domain simulations are performed with MATLAB software based on a Runge-Kutta algorithm with a sampling period of $\Delta t=.01 \mathrm{~s}$. A sampling period of $T_{\mathrm{s}}=.1 \mathrm{~s}$ is used for the control strategy. Future wave excitation force information is supposed to be perfectly known. Influence of how good wave excitation force is predicted, is out of topic in this paper. Unconstrained input control is presented in order to prove the proposed technique feasibility. After numerical investigation in regular wave analysis case, it have been found that a $14 \mathrm{~s}$ horizon time prediction seems to be required to provide accurate results. Constrained analysis is then performed for power production prediction at a given seestate. In this paper, we consider a $2.4 \mathrm{MW}$ generator power rating and a $3.75 \mathrm{~m} . \mathrm{s}^{-1}$ maximum linear relative velocity which impose a constraint of $640 \mathrm{kN}$ on the linear force that the power take-off could provide. In order to be more exhaustive, we have also considered a more restrictive control input of $530 \mathrm{kN}$.

Figure 4 shows numerical results in case of regular wave analysis. Considered wave amplitude and wave frequency are respectively $A=1 \mathrm{~m}$ and $\omega_{\mathrm{p}}=.85 \mathrm{rad} . \mathrm{s}^{-1}$. As expected from theoretical background [13], it appears that the relative 


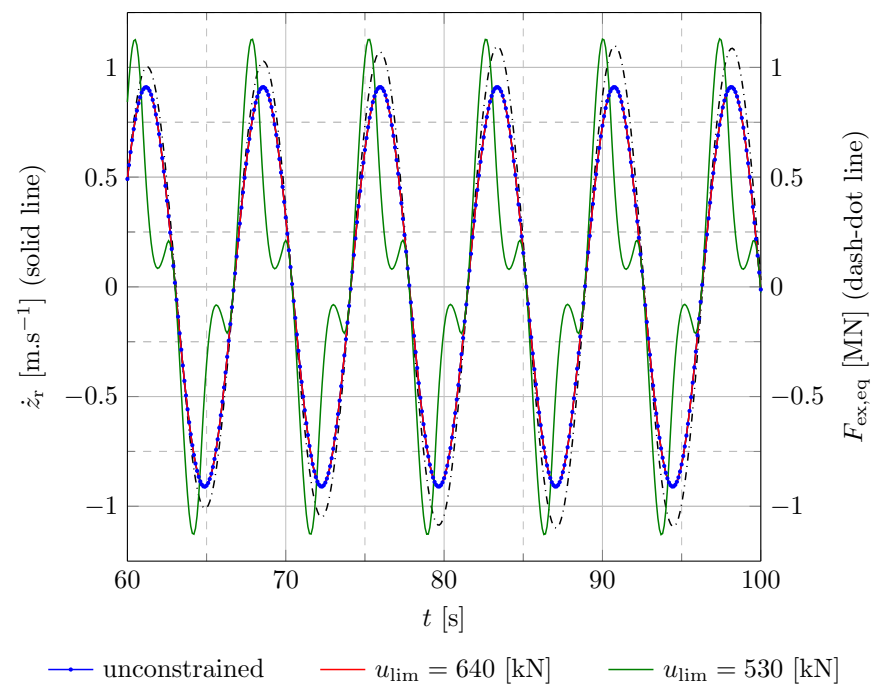

Fig. 4: Comparison between relative velocity and wave excitation force in case of regular wave analysis $(A=1 \mathrm{~m}$, $\left.\omega_{\mathrm{p}}=.85 \mathrm{rad} . \mathrm{s}^{-1}\right)$ for both uncontrained and constrained control strategy.

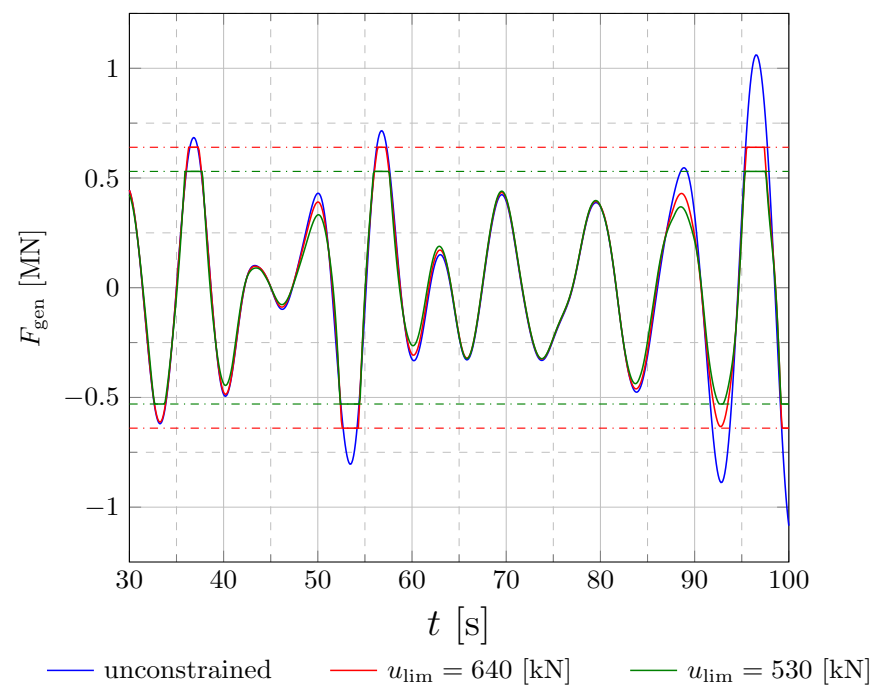

Fig. 5: Linear force due to the generator in case of irregular wave analysis.

velocity is in phase with the wave excitation force even when constrained control strategy is applied.

Figure 5 shows a more realistic example when the WEC is subjected to irregular wave conditions. Here, a JONSWAP's spectrum have been used which is characterised by a $2 \mathrm{~m}$ significant wave height, a $9 \mathrm{~s}$ peak period and, a 3.3 peak enhancement factor. Obviously it is clear that the control strategy is able to keep the PTO force within the desired control input constraints.

\section{CONCLUSION}

This paper dealt with the special case modelling and control of a self-reacting wave energy converter where reaction force is obtained using a damping plate. After presenting the full WEC model used for plant simulation, optimal WEC energy absorption in the time-domain was presented and the optimal control was formulated in a receding horizon fashion. In this paper, we proposed to use the "phenomenologically onebody equivalent model" description in the control problem formulation which reduces the model order and then is more appropriated for real-time application. Numerical results were presented for both regular and irregular wave analysis and also in unconstrained and constrained case in order to prove the proposed control strategy efficiency. Numerical results are encouraging and future works will investigate the use of this "phenomenologically one-body equivalent model" in case of a non-linear WEC model description.

\section{ACKNOWLEDGMENT}

This work was supported in part by the Fonds Unique Interministériel (France) - Project "EM Bilboquet", in part by Région Bretagne, and in part by Conseil Général du Finistère.

\section{REFERENCES}

[1] J. Cretel, A. W. Lewis, G. Lightbody, and G. P. Thomas, "An application of model predictive control to a wave energy point absorber," in Control Methodologies and Technology for Energy Efficiency, vol. 1, no. 1, Portugal, Mar. 2010, pp. 267-272.

[2] T. K. A. Brekken, "On Model Predictive Control for a point absorber Wave Energy Converter," in PowerTech, 2011 IEEE Trondheim, Trondheim, Norway, Jun. 2011, pp. 1-8.

[3] M. Richter, "Different Model Predictive Control Approaches for Controlling Point Absorber Wave Energy Converters," MSc thesis, Oregon State University, 2011.

[4] M. Richter, M. E. Magana, O. Sawodny, and T. K. A. Brekken, "Nonlinear Model Predictive Control of a Point Absorber Wave Energy Converter," IEEE Transactions on Sustainable Energy, vol. 4, no. 1, pp. 118-126, Jan. 2013.

[5] J. Falnes, "Wave-Energy Conversion Through Relative Motion Between Two Single-Mode Oscillating Bodies," Journal of Offshore Mechanics and Arctic Engineering, vol. 121, no. 1, pp. 32-38, Feb. 1999.

[6] Z. Yu and J. Falnes, "State-space modelling of a vertical cylinder in heave," Applied Ocean Research, vol. 17, no. 5, pp. 265-275, 1995.

[7] W. Cummins, "The impulse response function and ship motion," Tech. Rep., 1962.

[8] S. Olaya, J.-M. Bourgeot, and M. Benbouzid, "Modelling and Preliminary Studies for a Self-Reacting Point Absorber WEC," in International Conference on Green Energy 2014 (ICGE'2014), Sfax, Tunisa, Mar. 2014, pp. 14-19.

[9] T. Perez and T. I. Fossen, "A Matlab Toolbox for Parametric Identification of Radiation-Force Models of Ships and Offshore Structures," Modeling, Identification and Control: A Norwegian Research Bulletin, vol. 30, no. 1, pp. 1-15, 2009.

[10] J. Falnes, "On non-causal impulse response functions related to propagating water waves," Applied Ocean Research, vol. 17, no. 6, pp. 379-389, Dec. 1995.

[11] S. Olaya, J.-M. Bourgeot, and M. Benbouzid, "Hydrodynamic Coefficients and Wave Loads for a WEC Device in Heaving Mode," in Proceedings of the 2013 MTS/IEEE OCEANS, Bergen (Norway), Jun. 2013, pp. 1-8.

[12] S. Olaya, J.-M. Bourgeot, and M. Benbouzid, "Hydrodynamic Coefficient Computation for a Partially Submerged Wave Energy Converter," IEEE Journal of Oceanic Engineering, pp. 1-15 (in press), 2014.

[13] J. Falnes, Ocean Waves and Oscillating Systems - Linear Interactions Including Wave-Energy Extraction. Cambridge University Press, Apr. 2002.

[14] T. Perez and T. I. Fossen, "Practical aspects of frequencydomain identification of dynamic models of marine structures from hydrodynamic data," Ocean Engineering, vol. 38, no. 2-3, pp. 426-435, Feb. 2011. 